\title{
3D-volldigitalisierte Behandlungsplanung bei Lippen-Kiefer-Gaumenspalten (LKGS-3D)
}

Christiane Keil, Dominik Haim, Ines Zeidler-Rentzsch, Franz Tritschel, Bernhard Weiland, Olaf Müller, Thomas Treichel, Günter Lauer

Die Idealvorstellung eines vollständig digitalisierten Behandlungsalltags rückt mit fortschreitender technologischer und informationeller Entwicklung stetig näher an die Realität. Zu Beginn bestand lediglich die Möglichkeit einer elektronischen Patientenakte, hinzu kamen vielfältige Möglichkeiten der digitalen Bildgebung und wurden schließlich um das Ziel eines vollständigen digitalen Workflows ergänzt. Die Planung der interdisziplinären kieferorthopädischen / kieferchirurgischen Versorgung von Patienten mit Lippen-Kiefer-GaumenSpalten (LKGS) wurde bis vor kurzem am Universitätsklinikum Dresden noch hauptsächlich analog durchgeführt. Eine volldigitalisierte Behandlungsplanung unter Einbeziehung aller beteiligten Behandler fand nicht statt.

Ziel des Projektes war es deshalb, eine digitale Plattform zur interdisziplinären zahnmedizinischen Versorgung von LKGS-Patienten zu schaffen. Dazu wurde zuerst die bisher erforderliche Abdrucknahme mittels Alginat und die anschließende Herstellung eines Gipsmodells durch einen intraoralen 3D-Scan der Zahnbögen des Patienten abgelöst. Anhand des intraoralen 3D-Scans können nun die erforderlichen Trinkplatten mittels 3D-Druck erstellt werden. Zweiter Schritt war die Anfertigung von 3D-Aufnahmen der Weichteile des Gesichtes mittels eines extraoralen 3D-Scanners. Als dritter Schritt erfolgte die Anfertigung von Digitalen Volumentomografie (DVT)-Aufnahmen zur 3D-Darstellung des Schädelknochens und Kieferskeletts. Nach der Anfertigung wurden diese bildbasierten Datensätze zu einem „digitalen Zwilling" (virtuelles 3D-Modell aus DVT, intra- und extraoralen 3D-Scan) zusammengefasst, wodurch erstmalig ein umfassendes 3D-Modell des Mund-Kiefer-Raumes einschließlich wichtiger Informationen zum Kiefergelenk und der anliegenden Weichteile entstand. Dieses virtuelle Modell bildet jetzt die Grundlage für die Behandlungsplanung und die Planung der weiteren zahnmedizinischen und medizinischen Versorgung. Es konnte also im Projekt die komplette Digitalisierung der Diagnostik, die Etablierung einer Fusionsplattform und der Datenaustausch zwischen Uniklinik und privater Praxis umgesetzt werden.

Keywords: Digitalisierung, virtuelles 3D-Modell, MKG-Chirurgie, LKGS-Patienten 


\section{Einleitung}

Die Idealverstellung eines vollständig digitalisierten Behandlungsalltags rückt mit fortschreitender technologischer und informationeller Entwicklung stetig näher an die Realität. Zu Beginn bestand lediglich die Möglichkeit einer elektronischen Patientenakte, hinzu kamen vielfältige Möglichkeiten der digitalen Bildgebung und wurden schließlich um das Ziel eines vollständigen digitalen Workflows ergänzt. Die Planung der interdisziplinären kieferorthopädischen / kieferchirurgischen Versorgung von Patienten mit Lippen-Kiefer-Gaumen-Spalten (LKGS) wurde bis vor kurzem am Universitätsklinikum Dresden noch hauptsächlich analog durchgeführt. Eine volldigitalisierte Behandlungsplanung und -freigabe unter intersektoraler Einbeziehung aller beteiligten Behandler fand nicht statt. Neu entwickelte, digitale kieferorthopädische und -chirurgische Produkte, welche bis vor ein paar Jahren noch nicht denkbar waren, können aber den digitalen Workflow maßgeblich unterstützen. Intraorale und extraorale 3D-Scanner, 3DFotoaufnahmen und Ultraschalaufnahmen erstellen digitale Modelle, deren Daten zur Integration in den digitalen Workflow durch Softwareprozesse verarbeitet werden müssen. Um einen einfachen und strukturierten Zugriff auf die gesamten 3D-Daten zu gewährleisten, war die Idee entstanden, die anfallenden Daten und Unterlagen zu digitalisieren und in einer für diesen Zweck entwickelten Datenbank zu speichern, zu bearbeiten und zum Teil zu fusionieren. Die konsequente Verwendung von digitalen 3D-Analysen und der Verzicht auf die aufwändige Erstellung der Diagnostik- und Therapieplanung aus einer Kombination von Gipsmodellen und 2D-Daten (z.B. Röntgenbilder) führen zu einer fundamentalen und richtungsweisenden Veränderung des Behandlungsplanungsprozesses. Räumlich und monetär aufwendige Bearbeitungsprozesse werden eingespart und Behandlungspläne können rascher erstellt werden, da sich alle notwendigen Unterlagen gebündelt in einem System wiederfinden. Weiterhin wird die Strahlenbelastung der Patienten wesentlich verringert, da eine Doppeldiagnostik durch mehrmaliges Anfertigen von Röntgenaufnahmen, DVTs und CTs in verschiedenen Abteilungen vermieden wird. Die Kommunikation, der Austausch und die konsiliarische Zusammenarbeit zwischen den Behandlern erfolgt unter Verwendung von digitalen telemedizinischen Anwendungen zur Verbesserung der medizinischen Versorgung. 


\section{Zielstellung}

Ziel des aus Mitteln des Europäischen Fonds für Regionale Entwicklung (EFRE) und des Freistaates Sachsen in Höhe von bis zu 800.000 Euro geförderten Projektes ist die Nutzung digitaler Kommunikationswege zur interdisziplinären zahnmedizinischen Versorgung von LKGS-Patienten (Abbildung 1).

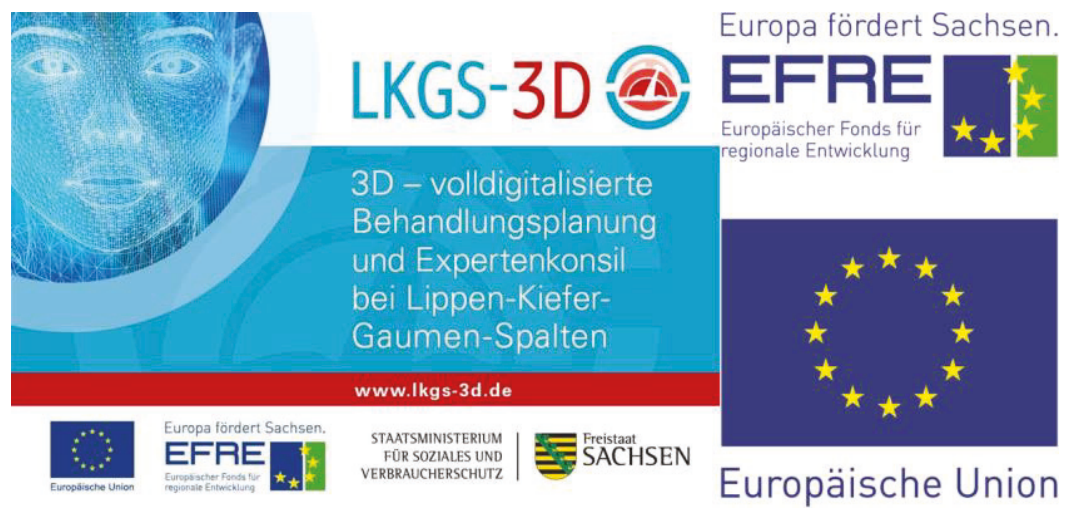

Abbildung 1: Logo des EFRE-Projektes "LKGS-3D"

Dazu wird die aktuell verwendete Kombination aus 2D-Daten (Röntgenaufnahmen, extraorale und intraorale Fotos) und Herstellung von Gipsmodellen in eine digitale Analyse überführt. Dies geschieht in mehreren Stufen.

- Zuerst wird die bisher erforderliche Abdrucknahme mittels Alginat und die anschließende Herstellung eines Gipsmodells durch einen intraoralen 3D-Scan der Zahnbögen des Patienten abgelöst. Anhand des intraoralen 3D-Scans können dann die erforderlichen Trinkplatten mittels 3D-Druck erstellt werden.

- Zweiter Schritt ist die Anfertigung von 3D-Aufnahmen der Weichteile des Gesichtes mittels eines extraoralen 3D-Scanners. Des Weiteren sollen die Weichteile der Lippe mittels Ultraschall untersucht und bildlich dargestellt werden.

- Als dritter Schritt erfolgt die Anfertigung von Digitalen Volumentomografie (DVT)-Aufnahmen zur 3D-Darstellung des Schädelknochens und Kieferskeletts.

Nach der Anfertigung werden diese bildbasierten Datensätze zu einem „digitalen Zwilling" (virtuelles 3D-Modell aus DVT, intra- und extraoralen 3D-Scan) zusammengefasst, wodurch erstmalig ein umfassendes 3D-Modell des Mund-Kiefer-Raumes einschließlich wichtiger Informationen zu den anliegenden Weichteilen entsteht. Dieses virtuelle 
Modell bildet die Grundlage der Behandlungsplanung der Patienten, der fachlichen Diskussion mit Konsilexperten sowie der Freigabe dieser Behandlungsplanung durch die Kostenträger (Abbildung 2).

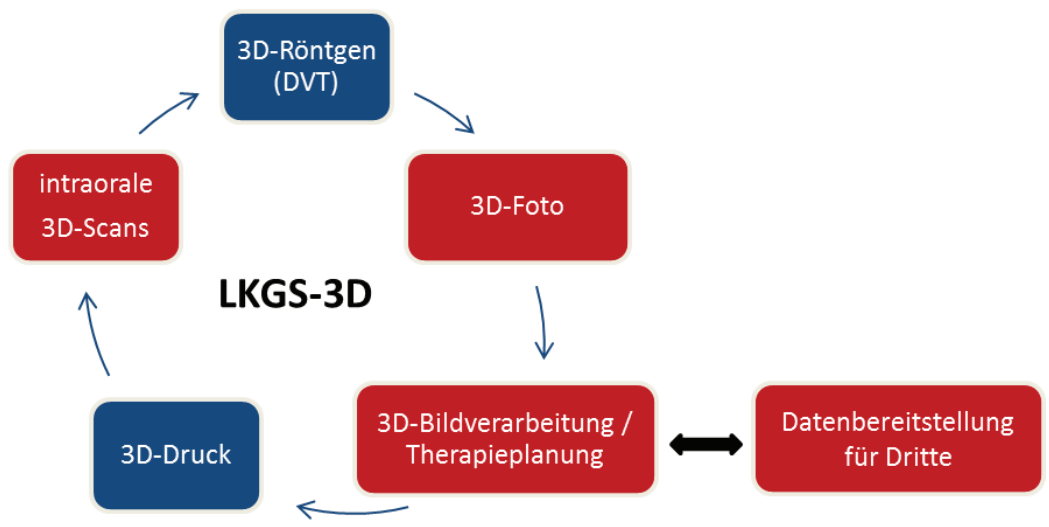

Abbildung 2: Darstellung des geplanten digitalen Prozesses für die Diagnostik- und Therapieplanung in der Behandlung von LKGS-Patienten. Rot markierte Felder sollen im Projekt bereitgestellt bzw. geschaffen werden.

Mit Hilfe der Plattform sollen drei Schwerpunkte innerhalb des Projektes bearbeitet werden:

1. Vollständige 3D-Digitalisierung des zahnärztlichen Planungs- und Freigabeprozesses

2. Optimierung der Behandlungsplanung und Verminderung der Strahlenbelastung bei Neugeborenen und Kindern mit LKGS

3. Intersektorale Vernetzung der kieferchirurgischen/ kieferorthopädischen Behandler in Sachsen.

\section{Umsetzung}

\section{Zu Schwerpunkt 1}

Neben Systemen für dreidimensionale DVT-Aufnahmen sind inzwischen zahlreiche intraorale wie extraorale Scanner auf dem Markt erhältlich. Eine durchgeführte Marktanalyse für Intraoralscanner ergab 16 momentan erhältliche Scansysteme, wovon der 
überwiegende Teil bereits puderfrei arbeitet, was Voraussetzung für die Anwendbarkeit in diesem Projekt ist. Der Einsatz von Intraoral-Scannern bei LKGS ist bisher nur wenig beschrieben. Es existiert lediglich eine Veröffentlichung / Arbeit, die beschreibt, dass mit Hilfe eines solchen Scanners die Gaumen von Kleinkindern mit LKGS zur Herstellung von Trinkplatten abgescannt werden konnten (Krey et al. 2018). Der von Krey et al. genutzte Intraoral-Scanner wurde im hier beschrieben Projekt an einem 9 Monate alten Kind mit LKGS getestet. Die Durchführung des Scans war möglich allerdings benötigte dieser mehr als 5 Minuten, was wiederum bei einem Kind von unter einem Jahr zu Schwierigkeiten führt (Stillliegen). Ein weiteres Problem offenbarte sich in der Tatsache, dass der Scanner sehr oft den Scan abbrach, da er die Strukturen nicht erkannte. Außerdem ist der Scanner sehr groß und lässt sich schwierig transportieren. Um dieses Transportproblem zu umgehen, wurde ein Wireless-Pod-Pen-Intraoralscanner für das Projekt beschafft. Um die Genauigkeit der Intraoralscans mit diesem Gerät im Vergleich zu herkömmlichen Alginatabdrücken bestimmen zu können, wurden zunächst freiwillige erwachsene Probanden gescannt. Auch hierbei konnte gezeigt werden, dass die digitale Abformung mit der klassischen Abformung in der Präzision vergleichbar ist. Der Einsatz des Intraoralscanners wurde im Anschluss daran intensiv an LKGS-Kindern im Alter von 6-9 Monaten während des operativen Lippen- bzw. Gaumenverschlusses (Ethikantragsnummer: SR + BO EK-43012020) getestet. Die Scans an den schlafenden Kindern waren völlig unkompliziert und problemlos. Es wurden während der höchstens 3-minütigen Scandauer alle Kieferstrukturen erkannt (siehe Abbildung 3A). Mit Hilfe dieses Scanners ist es außerdem möglich, die Digitalisierung der Weichteile, wie Lippe und Nase (Abbildung 3B) vorzunehmen, was wichtig für die Zusammensetzung eines 3D-Modells aus Intra- und Extraoralscans ist.

A

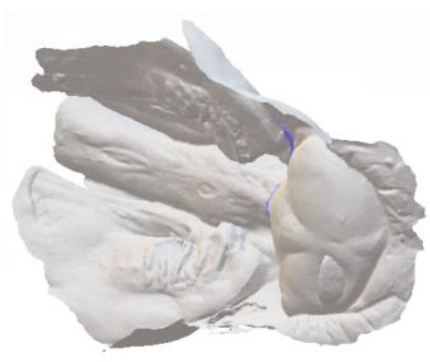

Abbildung 3: (A) Intraoralscan des Oberkiefers und (B) 3D-Scan der Oberlippe und der Nase eines 9 Monate alten Kindes mit LKGS

B

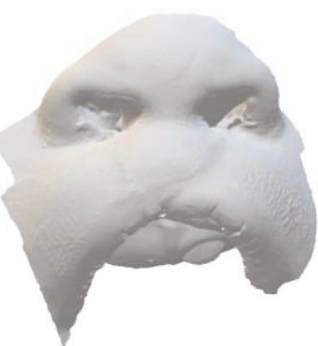


Die gewonnenen Intraoralscans wurden in einem ersten Pilotversuch dazu verwendet, sogenannte Trinkplatten zu erstellen. In Zusammenarbeit mit der Firma Biodentis $\mathrm{GmbH}$ aus Leipzig wurde zunächst die Trinkplatte anhand der zugesandten stl-Dateien digital entworfen und diese dann mittels CAD/CAM-Technik hergestellt (Abbildung 4).
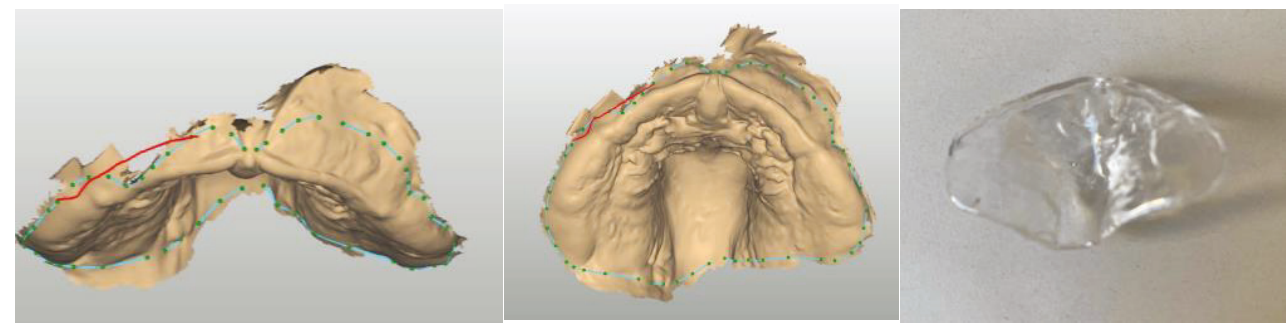

Abbildung 4: Labiale und palatinale Ansicht des Intraoralscans mit eingezeichnetem Entwurf der Trinkplatte (türkisene Linie) sowie daraus resultierende fertige Trinkplatte

Während der Anwendung des Intraoralscanners bei den Kindern wurde aber auch festgestellt, dass der Scanner für Kinder jünger als 6 Monate nicht geeignet ist, da der Scannerkopf dann zu groß ist und dadurch die kontaktfreie optische Oberflächenmesstechnik (Triangulation) nicht mehr angewendet werden kann (Abbildung 5).

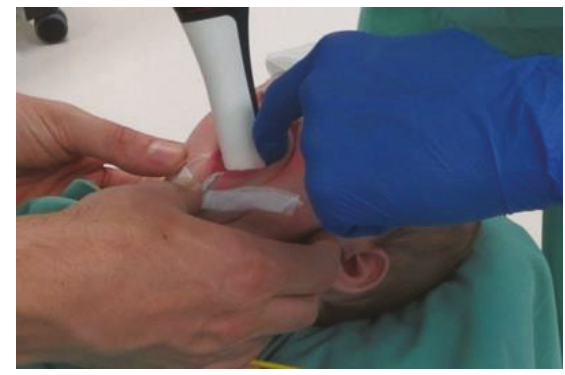

Abbildung 5: Anwendung des Intraoralscanners bei einem narkotisierten sechs Monate alten Kind mit LKGS

Um die Weichteile des Gesichts komplett digital darstellen zu können, eignen sich sogenannte Extraoralscanner. Laut Marktanalyse konnten für das Projekt fünf geeignete Systeme identifiziert werden. Auch hier wurde wieder das transportable System ausgewählt. Der Scan erfolgte schnell und problemlos. Das ausgewählte Gerät kann ohne Probleme bei LKGS-Kindern angewendet werden (Abbildung 6). 


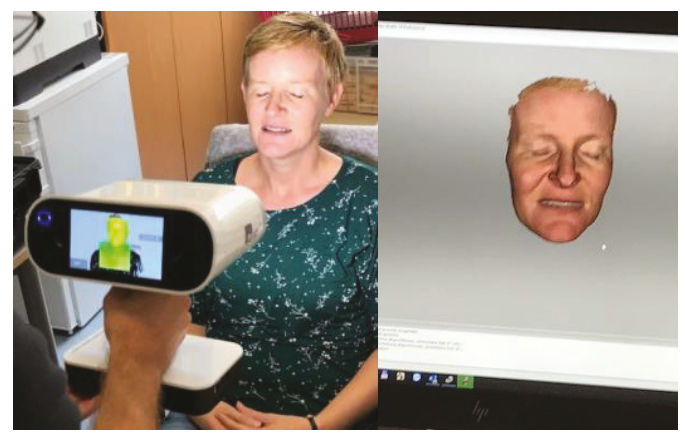

Abbildung 6: Anwendung des Extraoralscanners bei einer Probandin und digitale Rekonstruktion

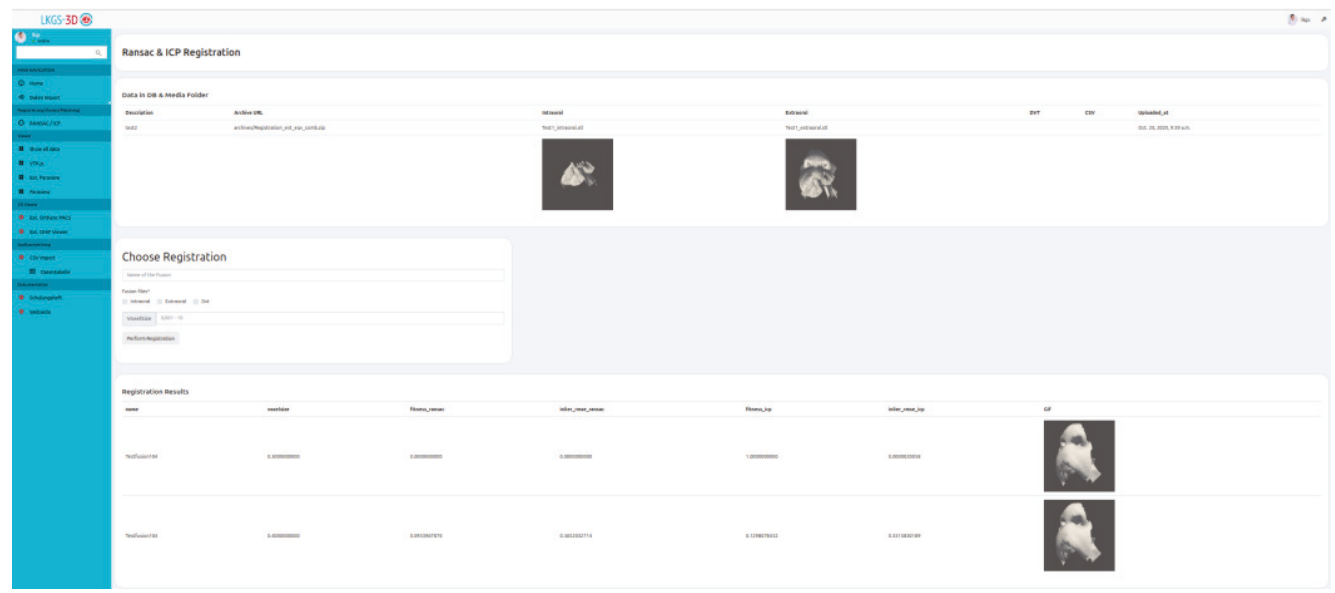

Abbildung 7: Screenshot der LKGS-3D Fusionsplattform mit Beispielfusionsbildern

Für die Umsetzung der volldigitalen Behandlungsplanung war es zunächst notwendig, den gesamten Behandlungsprozess vollständig zu ermitteln und zu hinterfragen. Von zentraler Bedeutung in diesem Prozess ist die Art und der Zeitpunkt der jeweils angewandten Diagnostik im Behandlungsverlauf sowie deren Dokumentation. Ferner wurden Anwender- bzw. Nutzerrollen und die damit verbundene Dokumentations- und Zugriffsrechte spezifiziert.

Für die Fusion verschiedener diagnostischer Unterlagen wurde eine neue Plattform geschaffen. Damit können je nach Bedarf sowohl Intra- und Extraoralscans als auch 3D-Röntgenaufnahmen zu sogenannten Fusionsmodellen zusammengefügt werden (Abbildung 7). 


\section{Zu Schwerpunkt 2}

Zweiter großer Schwerpunkt des Projektes beschäftigt sich mit Alternativen zur digitalen Volumentomographie (DVT) bzw. Computertomographie (CT). Das DVT nutzt Röntgenstrahlen als Grundlage des dreidimensionalen, bildgebenden Tomographie-Verfahrens. Für das Verfahren typisch sind eine isometrische Ortsauflösung im Volumen in allen drei Raumrichtungen sowie die Konzentration auf die Darstellung von Hochkontrast, d. h. auf Hartgewebe (Knochen). Es ist inzwischen bekannt, dass Knochen auch mit Hilfe von Ultraschall dargestellt werden können. Auch LKGS können bereits im Mutterleib mittels 2D- und 3D-Ultraschall diagnostiziert werden. Die Technik ist soweit ausgereift, dass bereits in der 14. Schwangerschaftswoche eine Diagnose erfolgen kann (Marginean et al. 2018). Des Weiteren konnte gezeigt werden, dass Ultraschall in der Lage ist, wichtige Merkmale des oralen orbitalen Lippenmuskels zu charakterisieren und diesen auch quantitative zu analysieren (de Korte et al. 2009). Für Patienten mit LKGS ist der diagnostische Einsatz einer Ultraschalluntersuchung ebenfalls geeignet. Die Gewebedimensionen lassen sich mit dieser Methode unproblematisch vermessen (van Hees et al. 2007).

Im Rahmen des Projektes erbrachte die Analyse der auf dem Markt zur Verfügung stehenden Ultraschallgeräte mit 3D-Ultrabreitbandtechnologie (Grundvoraussetzung für die Anwendung im Bereich Zahnmedizin) und spezifischen hochauflösenden Ultraschallsonden nur ein passendes Gerät. Dieses entsprechende Gerät wurde beschafft und konnte bereits mehrfach angewendet werden. Im Rahmen eines durch die Ethikkommission der TU Dresden begutachteten Pilotprojektes (BO-EK-336072020) werden momentan die Oberlippen von gesunden Probanden und LKGS-Patienten mittels Ultraschall untersucht, um die physiologische und pathologische Anatomie der Oberlippe bei LKGS-Patienten beschreiben zu können (Abbildung 8).

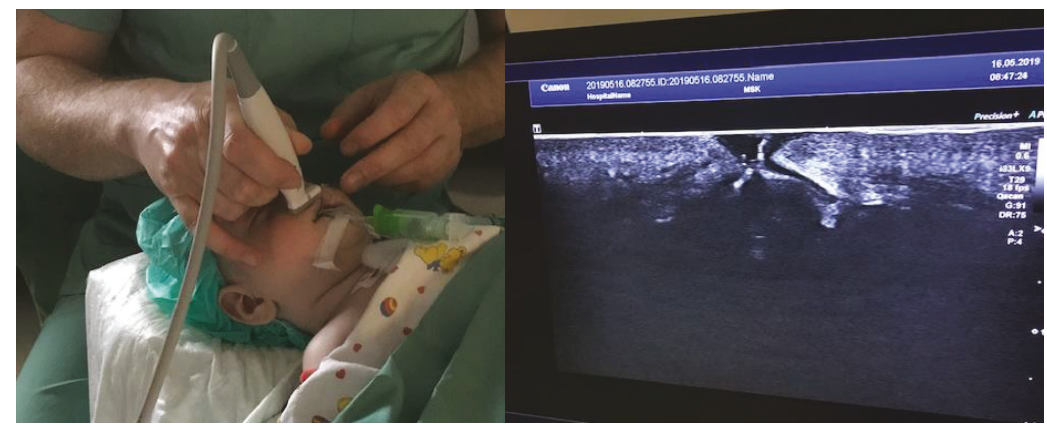

Abbildung 8: Ultraschall bei einem LKGS-Kind mittels spezifischer Hockeysticksonde 


\section{Zu Schwerpunkt 3}

Zur besseren Versorgung der Patienten ist es erforderlich, eine Vernetzung der überwiegend städtischen Versorgungszentren mit Praxen im ländlichen Raum herzustellen. Mit der CCS Telehealth Plattform Ostsachsen (THOS) stand zu Projektbeginn eine offene Kommunikationsplattform für medizinische Anwendungen zur Verfügung (Höhn). Auf Basis der durchgängigen Informationsbereitstellung als Grundvoraussetzung einer integrierten Versorgungsstruktur sollte damit eine bessere Zugänglichkeit zu medizinischen Leistungen, insbesondere in strukturschwachen ländlichen Bereichen, gewährleistet werden. Die Verwendung der THOS-Plattform für die Übertragung der Patientendaten konnte leider aus mehreren Gründen nicht umgesetzt werden. Zum einen waren die zu übertragenden Datenmengen sehr groß und zum anderen wurde ab Mitte 2020 die Plattform in der jetzigen Form nicht mehr von der Deutschen Telekom angeboten.

Um aber trotzdem eine Anbindung der ambulanten Praxen vollziehen zu können, wurde der Vorschlag des Projektpartners Computer konkret AG umgesetzt. Dieser Vorschlag sieht vor, das Computerprogramm Ivoris analyze für den Datenaustausch zu nutzen (Computerkonkret). Der Import der Daten und der Fusionsmodelle erfolgt ohne Probleme (Abbildung 9).

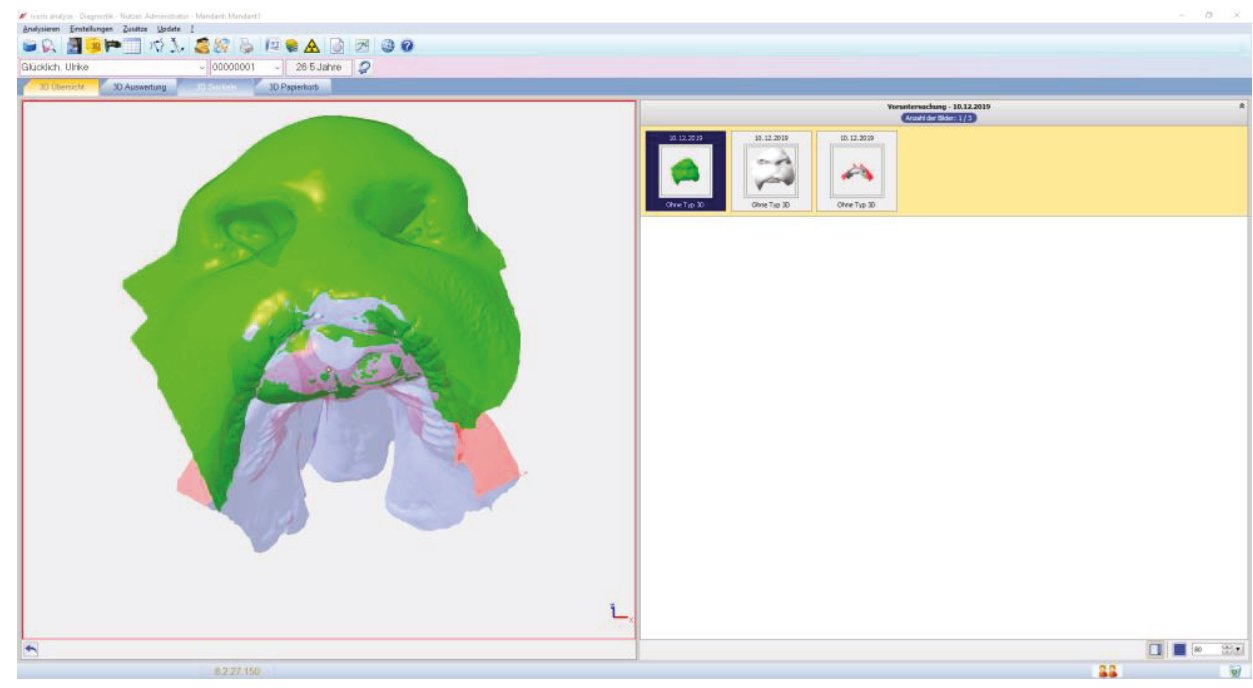

Abbildung 9: Screenshot der Software "Ivoris analyze" mit Beispielfusionsbildern 
Für den Datenaustausch mit den ambulanten Praxen werden alle relevanten Daten zu sogenannten Paketen zusammengefasst. Die Kommunikation ist in beide Richtungen und gleichzeitig auch zwischen mehr als zwei Kommunikationspartnern möglich. Der Inhalt von Paketen kann individuell zusammengestellt und weitgehend automatisch in die Software importiert als auch aus der Software exportiert werden.

\section{Literaturverzeichnis}

Computerkonkret. ivoris - Dental Software Power. https://www.ivoris.de/diagnostik.html, veröffentlicht 2018, aufgerufen am 16.02.2021.

de Korte, CL, van Hees, N, Lopata, RG, Weijers, G, Katsaros, C \& Thijssen, JM (2009): Quantitative assessment of oral orbicular muscle deformation after cleft lip reconstruction: an ultrasound elastography study. In: IEEE Trans Med Imaging, 28 (8), 1217-1222.

Höhn, T. CCS Telehealth Ostsachsen (THOS) - das größte Telemedizin-Vorhaben Deutschlands. https://www.vdz.org/digitalekommune/ccs-telehealth-ostsachsen-thos-das-groesste-telemedizin-vorhaben-deutschlands, veröffentlicht 2018, aufgerufen am 16.02.2021.

Krey, KF, Ratzmann, A, Metelmann, PH, Hartmann, M, Ruge, S \& Kordass, B (2018): Fully digital workflow for presurgical orthodontic plate in cleft lip and palate patients. In: Int J Comput Dent, 21 (3), 251-259.

Marginean, C, Sasarean, V, Marginean, CO, Melit, LE \& Marginean, MO (2018): Prenatal diagnosis of cleft lip and cleft lip palate - a case series. In: Med Ultrason, 20 (4), 531-535.

van Hees, NJ, Thijssen, JM, Huyskens, RW, Weijers, G, Nillesen, MM, de Korte, CL \& Katsaros, C (2007): Quantitative ultrasound imaging of healthy and reconstructed cleft lip: a feasibility study. In: Cleft Palate Craniofac J, 44 (3), 261-268.

\section{Kontakt}

PD Dr. rer. nat. habil. Christiane Keil

Dr. med. Dominik Haim

Dr. rer. nat. Ines Zeidler-Rentzsch

Franz Tritschel

Dr. med. dent. Bernhard Weiland

Prof. Dr. med. Dr. med. dent. Günter Lauer

Klinik und Poliklinik für Mund-Kiefer-

Gesichtschirurgie der TU Dresden

Fetscherstr. 74

01307 Dresden

www.uniklinikum-dresden.de/mkg
Dr. Olaf Müller

Carus Consilium Sachsen GmbH

Fetscherstr. 74

01307 Dresden

www.carusconsilium.de

Thomas Treichel

Computer konkret AG

Theodor-Körner-Str. 6

08223 Falkenstein

www.computer-konkret.de 